\title{
INSTABILITY OF MARGINALLY STABLE STREAMWISE VARYING SHEAR FLOW TO LONG ROSSBY WAVES*
}

\author{
DANIEL HODYSS ${ }^{\dagger}$ AND TERRENCE R. NATHAN $¥$
}

\begin{abstract}
The instability of streamwise varying shear flow that is marginally stable to long Rossby waves is examined. Both Hamiltonian and non-Hamiltonian flows are considered within the framework of coupled wave instability (CWI). The CWI is shown to be mediated by a 'physical' wave and a 'virtual' wave. The physical/virtual wave model concisely describes the differences between the instabilities that develop on locally supercritical flow and those that develop on globally subcritical flow. Globally subcritical Hamiltonian flows are proven to be stable. In contrast, nonHamiltonian flows may be unstable in both locally supercritical and globally subcritical regimes. In locally supercritical flow, the CWI grows via wave-resonance between the physical/virtual wave pair and is highly localized to the supercritical region. In contrast, in globally subcritical flow, the CWI grows via pseudomomentum extraction from the background flow and either radiates away or remains attached to the streamwise variation in the flow.
\end{abstract}

Key words. linear instability, coupled wave, long Rossby waves, streamwise varying

MSC subject classifications. 76B65, 76E09, 76E15, 76E20

\section{Introduction}

Rossby waves are ubiquitous. They may exist in any medium that possesses a background potential vorticity (PV) gradient, including Earth's atmosphere and oceans ([1], [2]), the Sun's interior ([3], [4]), and spiral galaxies ([5], [6]). Consequently, the literature on Rossby waves is vast, with most of it occupied by studies examining their dynamics in media for which the background PV gradient is streamwise uniform (e.g., [7], [8]). Relatively few studies have examined the dynamics of Rossby waves in media for which the background PV gradient is streamwise varying, despite such media often being in closer aligment with observations.

In Earth's atmosphere, for example, streamwise varying shear flow is commonplace, owing in large part to longitudinal asymmetries in bottom topography and diabatic heating. The streamwise variations produced by these forcings play a significant role in both the genesis and regional structure of low frequency (LF) Rossby waves (e.g., [9], [10]). Yet, despite these findings, fundamental questions remain. For example, are there differences between the instabilities that develop on Hamiltonian versus non-Hamiltonian flows? How do variations in the local stability properties of the flow control the emergence of Rossby wave instabilities? Is the instability mechanism that traditionally operates in streamwise uniform flow, i.e., wave-resonance (e.g.,[11]), also operating in streamwise varying flow? If not, are there other, possibly new, instability mechanisms operating?

To provide a framwork for addressing these questions first requires a brief review of the stability criteria associated with streamwise uniform background flow. To anchor the discussion, consider the simplest case of a rotating, two-dimensional incompressible fluid confined to a channel of width $2 L$. For this fluid system the necessary condition for instability of normal mode disturbances takes the following form

*Received: February 21, 2007; accepted (in revised version): August 1, 2007. Communicated by Paul Milewski.

${ }^{\dagger}$ Naval Research Laboratory, 7 Grace Hopper Avenue, Monterey, CA 93943, USA (daniel.hodyss@ nrlmry.navy.mil).

${ }^{\ddagger}$ Atmospheric Science Program, Department of Land, Air, and Water Resources, University of California, Davis, California 95616, USA (trnathan@ucdavis.edu). 
(e.g., [12]):

$$
\omega_{i} \int_{-L}^{L} \frac{k|H(y)|^{2}}{|k U(y)-\omega|^{2}} \frac{d Q(y)}{d y} d y=\omega_{i} I=0,
$$

where $y$ is the cross-stream direction, $\omega=\omega_{r}+i \omega_{i}$ is the complex eigenfrequency, $k$ is the streamwise (zonal) wavenumber, $H(y)$ is the cross-stream disturbance structure, and $d Q / d y$ is the absolute vorticity gradient of the background flow. Based on (1.1), we define five stability regimes: (1) unstable $\left(\omega_{i}>0, I=0\right)$, where $I=0$ requires that the absolute vorticity gradient change sign somewhere in the domain; (2) deeply stable $\left(\omega_{i}=0, I=O(1)\right) ;(3)$ marginally stable $\left(\omega_{i}=0, I=0\right) ;(4)$ supercritical $\left(\omega_{i}=O(\varepsilon)>\right.$ $0, I=0)$, where $\varepsilon \ll 1$; and (5) subcritical $\left(\omega_{i}=0, I=O(\epsilon)\right)$. The supercritical and subcritical stability regimes result from small, i.e., $O(\epsilon)$, departures of the background flow from marginal stability, and are the focus of this article.

The stability regimes that we have defined for streamwise uniform flow will be applied locally to characterize streamwise varying flow. In particular, we will show that for streamwise varying shear flow, there are sharp differences in the Rossby wave instabilities that develop on (1) deeply stable versus marginally stable flows and on (2) locally supercritical verus globally subcritical flows. These differences will be shown to manifest themselves in the stability mechanism, stability criteria, and disturbance structure.

To examine the connection between the streamwise variations in the background flow and the Rossby wave instabilities that subsequently develop, we will build our analysis on the foundation set by Hodyss and Nathan ([13]; hereafter HN06), who analytically examined the local stability of streamwise varying media to a broad class of long waves. Hodyss and Nathan ([14]) [hereafter HN07] subsequently showed, using a quasigeostrophic (QG), barotropic model, that HN06's generalized stability analysis applies to long Rossby waves that develop on streamwise varying background flows that are everywhere deeply stable. They obtained the striking result that streamwise varying flow that is deeply stable at every streamwise location may nonetheless be unstable to exponentially amplifying disturbances.

The deeply stable flow regime examined by HN07 may not always be met in the atmosphere. Stone $([15])$, for example, has shown that on seasonal time scales the extratropical zonal flow is marginally stable to synoptic-scale Rossby waves. It is therefore reasonable to expect that at certain times during the seasonal evolution of the atmosphere the extratropical zonal flow may also be marginally stable to long Rossby waves. With such times in mind, we examine the extent to which the results obtained by HN07 apply to streamwise varying background flows that are marginally stable.

To carry out the marginal stability analysis, we will utilize the linearized form of the long, low frequency Rossby wave equation derived by Hodyss and Nathan ([16]; hereafter HN04). That equation derives from the classic two-layer model for baroclinic instability, wherein the streamwise variations in the background flow are chosen to be asymptotically small and to vary on the same scale as the long-wave. Although HNO4 carried out some linear stability calculations, their focus was on the mechanisms that excite nonlinear instabilities. Here we show that the linear instability of the streamwise varying flow examined by HNO4 can be explained as a coupled wave instability (CWI). More importantly, the CWI framework clearly exposes the fundamental differences in stability criteria between marginally stable and 
deeply stable streamwise varying shear flows and between supercritical and subcritical flow regimes.

The paper is organized as follows. In Section 2 the model is briefly described, and in Section 3 the long Rossby wave equation derived by HN04 is reviewed to the extent necessary to highlight the physical and mathematical setting; two new conservation laws are also introduced. Stability criteria for marginally stable Hamiltonian flows are derived in Section 4 and compared to the stability criteria derived in HN06 for deeply stable Hamiltonian flows. In Section 5, which considers non-Hamiltonian flow, a complex WKB analysis is carried out to obtain an expression for the global mode frequency and structure. The conclusions are given in Section 6 .

\section{The model}

We will employ the classic two-layer quasigeostrophic (QG) model ([12]). To anchor the discussion we formulate the model in a geophysical context. We emphasize, however, that the long-wave equation to be derived below relies only on the assumptions of quasigeostrophy and marginal stability, not on the two-layer fluid configuration. In fact, it can be shown that under the assumptions of quasigeostrophy and marginal stability, long wave equations can be derived for both barotropic and continuous QG models that are equivalent to the long wave equation derived here.

The two-layer QG model consists of two layers of homogeneous, incompressible fluids of different densities in a gravitationally stable configuration (less dense fluid overlies more dense fluid). The fluid system is rotating with angular speed $\Omega$ about the vertical axis and is bounded above and below by flat, rigid boundaries and is confined to a streamwise infinite channel. The channel walls, which are rigid and vertical, are a distance $2 L$ apart in the cross-stream direction. The beta-plane approximation is used to account for the Earth's sphericity, i.e., $2 \Omega=f_{0}+\beta^{*} y^{*}$, where $f_{0}=2 \Omega \sin \theta_{0}$ is the Coriolis parameter and $\beta^{*}$ is the dimensional, northward gradient of $f$. Both $f_{0}$ and $\beta^{*}$ are evaluated at the central latitude, $\theta_{0}=45^{\circ}$.

The non-dimensional equations governing the evolution of the model atmosphere are:

$$
\frac{\partial q_{n}}{\partial t}+J\left(\psi_{n}, q_{n}\right)=G_{n}
$$

where the QG potential vorticity $(\mathrm{PV})$ is defined as

$$
q_{n}=\nabla^{2} \psi_{n}+\beta y+F(-1)^{n}\left(\psi_{1}-\psi_{2}\right)
$$

The subscript $n=1 \quad(\mathrm{n}=2)$ denotes variables defined in the upper (lower) layer, $J(a, b) \equiv a_{x} b_{y}-a_{y} b_{x}$, and $\nabla^{2}=\partial_{x x}+\partial_{y y}$. The streamwise and cross-stream directions are denoted by $x$ and $y$, and $t$ denotes time. $\psi_{n}$ is the geostrophic streamfunction, where

$$
U_{n}=-\frac{\partial \psi_{n}}{\partial y}, V_{n}=\frac{\partial \psi_{n}}{\partial x},
$$

are the streamwise (zonal) and cross-stream (meridional) velocities. $G_{n}$ is an external PV source that drives the background flow. In deriving (2.1), the scales $L, D, U$, $U L, D U / f_{0} L$ and $L / U$ have been used to non-dimensionalize the length and depth of each layer, horizontal velocities, streamfunction and time, respectively. The nondimensional planetary vorticity gradient, $\beta$, and internal rotational Froude number, $F$, are: $\beta=\beta^{*} L^{2} / U$ and $F=f_{0}^{2} L^{2} / D(g \Delta \rho / \rho)$, where $g$ is the gravitational acceleration, 
$\Delta \rho$ is the difference in density between the two fluid layers, and $\rho$ is the mean density.

In the streamwise limits, $x \rightarrow \pm \infty$, the background flow is streamwise uniform and the disturbance field vanishes. For the cross-stream boundary conditions we require that the flow normal to the channel sidewalls, at $y= \pm 1$, vanish.

\section{Long wave equation}

In this section we present the asymptotic deveopment of the long wave equation and use a coupled wave framework to derive conservations laws that provide constraints on the structure and growth of the wave field.

3.1. Asymptotic development. The total streamfunction field is partitioned into a background flow and a disturbance field:

$$
\psi_{n}(x, y, t)=\underset{\text { Background Flow }}{\Psi_{n}(x, y)}+\underset{\text { Disturbance Field }}{\phi_{n}^{\prime}(x, y, t)} .
$$

The background flow is chosen to be a steady, vertically sheared current that varies in the streamwise and cross-stream directions. The background flow is driven by the external PV source, $G_{n}$, and satisfies

$$
J\left(\Psi_{n}, Q_{n}\right)=G_{n},
$$

where $Q_{n}$ is the background PV. Insertion of (3.1) into (2.1), and subsequently subtracting (3.2), yields the following equation for the disturbance field:

$$
\frac{\partial q_{n}^{\prime}}{\partial t}+J\left(\phi_{n}^{\prime}, Q_{n}\right)+J\left(\Psi_{n}, q_{n}^{\prime}\right)+J\left(\phi_{n}^{\prime}, q_{n}^{\prime}\right)=0,
$$

where $q_{n}^{\prime}$ is the disturbance PV. To isolate the long, low frequency motions in (3.3), we introduce the long zonal scale $X=\epsilon^{1 / 2} x$ and long time scale $T=\epsilon t$, where $\epsilon \ll 1$. For the background flow we choose

$$
\Psi_{n}(X, y, T)=-\int_{-1}^{y}\left(U_{n}^{(0)}\left(y^{\prime}\right)+\epsilon U_{n}^{(1)}\left(X, y^{\prime}\right)\right) d y^{\prime},
$$

which has been partitioned into two parts. As in ([7]), the first part is an $\mathrm{O}(1)$ streamwise uniform current that is chosen marginally stable to long, low frequency waves. In the two-layer, QG model considered here, the point of marginal stability in the long, low-frequency limit requires that the normal mode disturbance and streamwise uniform flow together satisfy

$$
I_{Q G}=\sum_{n=1}^{2} \int_{-1}^{1} \frac{H_{n}^{2}}{U_{n}^{(0) 2}} \frac{d Q_{n}^{(0)}}{d y} d y=0,
$$

where $H_{n}(y)$ is the cross-stream normal mode structure. The second part of the background flow in (3.4) measures the small streamwise varying perturbation to the $\mathrm{O}(1)$ streamwise uniform flow. If we were to evaluate (3.5) locally with the total flow (3.4), we would find that the small streamwise perturbation produces locally subcritical $\left(\omega_{i}=0\right.$ and $\left.I_{Q G}=O(\epsilon)\right)$ and supercritical $\left(\omega_{i}=O(\epsilon)>0\right.$ and $\left.I_{Q G}=0\right)$ regions in the flow. If $U_{n}^{(1)}$ is chosen constant in the streamwise direction, then the background flow becomes identical to that used by Helfrich and Pedlosky ([7], [8]) in their studies of 
isolated anomalies in baroclinic zonal currents.

Given the background flow (3.4), the long, low frequency Rossby wave equation is obtained by expanding the disturbance streamfunction field in the asymptotic series,

$$
\phi_{n}^{\prime}=\epsilon \phi_{n}^{(0)}+\epsilon^{3 / 2} \phi_{n}^{(1)}+\ldots
$$

Insertion of (3.4) and (3.6) into (3.3) yields a sequence of linear problems in powers of $\epsilon$. By demanding that at each order in $\epsilon$ the solutions remain orthogonal to the homogenous adjoint solution, we obtain, to $\mathrm{O}\left(\epsilon^{3 / 2}\right)$, the following solution for the disturbance field:

$$
\phi_{n}^{\prime}(X, y, T)=\epsilon A(X, T) H_{n}(y)+\epsilon^{3 / 2} \Phi(X, T) f_{n}(y),
$$

where $A(X, T)$ is the amplitude, $H(y)$ is the meridional structure, and $\Phi(X, T) f_{n}(y)$ is the vertical and horizontal phase shift of the Rossby wave field. As shown in ([7]), the small phase shift term, $\epsilon^{3 / 2} \Phi(X, T) f_{n}(y)$, and the meridional structure, $H(y)$, do not have to be calculated explicitly in order to obtain generalized solutions for the Rossby wave amplitude. It will suffice to show that the meridional structure of the Rossby wave field satisfies

$$
\begin{gathered}
\frac{d^{2} H_{n}}{d y^{2}}+F(-1)^{n}\left(H_{1}-H_{2}\right)=\kappa_{n} H_{n}, \\
H_{n}=0, y=-1,1,
\end{gathered}
$$

where $\kappa_{n}=-Q_{n y}^{(0)} / U_{n}^{(0)}$. In the special case where the background flow $U_{n}^{0}$ is constant, the meridional structure function is defined by sinusoidal waves (see Appendix A).

The Rossby wave amplitude, $A(X, T)$, satisfies a variable-coefficient Boussinesq equation

$$
m_{1} \frac{\partial^{2} A}{\partial T^{2}}+\frac{\partial}{\partial X}\left[m_{2} \frac{\partial^{3} A}{\partial X^{3}}+m_{3}(X) \frac{\partial A}{\partial X}+m_{4} A \frac{\partial A}{\partial X}+m_{5}(X) A\right]=0,
$$

where $A \rightarrow 0$ as $|X| \rightarrow \infty$ has been used. The coefficients $m_{j}(j=1-5)$, which are discussed in [7] and [16], are defined in Appendix B.

Before proceeding to the analysis of (3.10), it is instructive to first show how the terms in the amplitude equation are related to the original terms in the disturbance PV Equ. (3.3):

$$
\begin{gathered}
U_{n}^{(0)} \frac{\partial q_{n}^{(1)}}{\partial X} \Longrightarrow \frac{\partial}{\partial X}\left(m_{2} \frac{\partial^{3} A}{\partial X^{3}}\right) \\
U_{n}^{(1)} \frac{\partial q_{n}^{(1)}}{\partial X}+\frac{\partial Q_{n}^{(1)}}{\partial y} \frac{\partial \phi_{n}^{(1)}}{\partial X} \Longrightarrow \frac{\partial}{\partial X}\left(m_{3}(X) \frac{\partial A}{\partial X}\right) \\
J\left(\phi_{n}^{(1)}, q_{n}^{(1)}\right) \Longrightarrow \frac{\partial}{\partial X}\left(m_{4} A \frac{\partial A}{\partial X}\right) \\
V_{n}^{(1)} \frac{\partial q_{n}^{(1)}}{\partial y}-\frac{\partial Q_{n}^{(1)}}{\partial X} \frac{\partial \phi_{n}^{(1)}}{\partial y} \Longrightarrow \frac{\partial}{\partial X}\left(m_{5}(X) A\right) .
\end{gathered}
$$


Equations (3.11) and (3.13), which are the linear dispersion and nonlinear terms, arise from the advection of the Rossby wave by the streamwise uniform flow and from the advection of the Rossby wave by itself. Equations (3.12) and (3.14), which depend on streamwise variations in the background flow through the coefficients $m_{3}(X)$ and $m_{5}(X)$, describe the phase speed modulation and local growth of the Rossby wave field. For the subsequent analysis, it suffices to note that $m_{2}>0$ and that $m_{5}=0$ in streamwise uniform flow (see Appendix B).

It will prove useful to write (3.10) in canonical form by the following re-scaling:

$$
\begin{aligned}
\frac{\partial}{\partial X} & \rightarrow \frac{1}{m_{2}^{\frac{1}{4}}} \frac{\partial}{\partial X}, \\
\frac{\partial}{\partial T} & \rightarrow \frac{1}{m_{1}^{\frac{1}{2}}} \frac{\partial}{\partial T}, \\
A & \rightarrow \frac{m_{2}^{\frac{1}{2}}}{m_{4}} A, \\
m_{3} & \rightarrow \frac{1}{m_{2}^{\frac{1}{2}}} m_{3}, \\
m_{5} & \rightarrow \frac{1}{m_{2}^{\frac{1}{4}}} m_{5},
\end{aligned}
$$

which allows the evolution equation to be written in only two parameters, $m_{3}(X)$ and $m_{5}(X)$ :

$$
\frac{\partial^{2} A}{\partial T^{2}}+\frac{\partial}{\partial X}\left[\frac{\partial^{3} A}{\partial X^{3}}+m_{3}(X) \frac{\partial A}{\partial X}+A \frac{\partial A}{\partial X}+m_{5}(X) A\right]=0 .
$$

Without loss of generality, the analysis below is based on this simplified form of the evolution equation.

3.2. Coupled wave framework. The properties of the instabilities that arise in (3.20) are most easily identified by introducing two coupled waves, $A(X, T)$ and $B(X, T)$, such that (3.20) may be written as two coupled equations, viz.,

$$
\begin{gathered}
\frac{\partial A}{\partial T}=\frac{\partial B}{\partial X} \\
\frac{\partial B}{\partial T}=-\frac{\partial^{3} A}{\partial X^{3}}-m_{3}(X) \frac{\partial A}{\partial X}-A \frac{\partial A}{\partial X}-m_{5}(X) A .
\end{gathered}
$$

Equ. (3.21) is an advection equation whereas (3.22) is related to the variable coefficient Korteweg-deVries equation examined by HN07 in their study of long Rossby waves in streamwise varying, deeply stable flow. If steady state conditions are assumed, then (3.21) and (3.22) reduce to the steady state form of the equation examined in HN07.

Because wave $A$ is the solution to (3.20), it is measurable in a physical system 
and thus will be referred to as the 'physical' wave. In contrast, wave $B$ is an artifact of our CWI conceptual framework and thus will be referred to as the 'virtual' wave. The introduction of the virtual wave $B$ serves two purposes: (1) it makes clear the connection between the marginally stable and deeply stable cases; (2) it facilitates the derivation and interpretation of the conservation laws to be derived below.

Waves that satisfy (3.21) and (3.22) also satisfy the following conservation laws:

$$
\begin{gathered}
\frac{d}{d T} \int_{-\infty}^{\infty} A d X=0 \\
\frac{d}{d T} \int_{-\infty}^{\infty} B d X=\int_{-\infty}^{\infty}\left(\frac{d m_{3}}{d X}-m_{5}\right) A d X,
\end{gathered}
$$

where we have assumed that $B \rightarrow 0$ as $|X| \rightarrow \infty$. Conservation law (3.23) is extremely restrictive; it guarantees that all exponentially growing long Rossby waves have oscillatory structure. This is in sharp contrast to the deeply stable flow examined in HN07, where it was shown that oscillatory as well as non-oscillatory wave structures were possible.

A conservation equation for pseudomomentum can also be obtained from (3.21) and (3.22):

$$
\frac{d}{d T} \int_{-\infty}^{\infty} A B d X=\int_{-\infty}^{\infty} S(X) A^{2} d X
$$

where

$$
S(X)=\frac{1}{2}\left(\frac{d m_{3}}{d X}-2 m_{5}\right) .
$$

We will find it useful to refer to $S(X)$ as a local pseudomomentum 'source.' Physical/virtual wave pairs that have positive pseudomomentum grow where the local pseudomomentum source is positive $(S>0)$, and wave pairs that have negative pseudomomentum grow where the local pseudomomentum source is negative $(S<0)$. Additionally, as shown below, in streamwise uniform flow, $S=0$ yields pseudomomentum conservation. When pseudomentum is conserved, $A$ and $B$ must be orthogonal for instability. Lastly, it is interesting to note that the nonlinear term plays no explicit role in the growth and decay of these wave activity measures [(3.23),(3.24), and (3.25)].

Based on the pseudomementum Equ. (3.25), we have identified two distinct mechanisms for instability. The first mechanism is wave-resonance, where $A$ and $B$ are locally configured such that the pseudomomentum vanishes, which is the traditional mechanism that operates in streamwise uniform flow. The second mechanism is pseudomomentum extraction, where $A$ and $B$ are locally configured to have the same signed pseudomomentum as the pseudomomentum source, which is a new mechanism that operates only in streamwise varying flow. HN07 found that pseudomomentum extraction is the sole mechanism for growth found in deeply stable flow.

\section{Hamiltonian stability analysis}

In the following analysis we will focus on the behavior of the linear instabilities in the coupled amplitude equations (3.21) and (3.22) by neglecting the nonlinear term. However, we note that the nonlinear term may be incorporated by the simple addition 
of the appropriate term in the Hamiltonian density.

Equations (3.21) and (3.22) constitute a Hamiltonian system, provided

$$
m_{5}=\frac{d m_{3}}{d X}
$$

This condition is identical to the Hamiltonian condition derived in HN06 and HN07, a fact that may not be too surprising given that (3.22) is, as discussed above, directly related to the equation governing the linear dynamics of long Rossby waves in deeply stable flow. As shown in $\mathrm{HN} 07$, all background flows forced by topographic variations, and a well-defined class of external source functions $G_{n}$, satisfy an equation with the form of (4.1).

Given (4.1), the linearized amplitude equations can be written as a non-canonical Hamiltonian system of the form:

$$
\frac{\partial}{\partial T}\left[\begin{array}{l}
A \\
B
\end{array}\right]=\mathbf{J}\left[\begin{array}{l}
\delta H / \delta A \\
\delta H / \delta B
\end{array}\right]
$$

where the symplectic operator $\mathbf{J}$ is defined as

$$
\mathbf{J} \equiv\left[\begin{array}{cc}
0 & -\partial / \partial X \\
-\partial / \partial X & 0
\end{array}\right]
$$

and the Hamiltonian is defined as

$$
H=\frac{1}{2} \int_{-\infty}^{\infty}\left[m_{3} A^{2}-\left(\frac{\partial A}{\partial X}\right)^{2}-B^{2}\right] d X .
$$

Following the procedure in HN06, several necessary conditions for the global instability of the streamwise varying flow can be obtained by application of Noether's theorem to the Hamiltonian form of the amplitude evolution equation. For example, if the Hamiltonian (4.4) possesses translational symmetry in $\alpha$, a functional $\Pi$ is time invariant, provided

$$
\frac{\partial}{\partial \alpha}\left[\begin{array}{l}
A \\
B
\end{array}\right]=-\mathbf{J}\left[\begin{array}{l}
\delta \Pi / \delta A \\
\delta \Pi / \delta B
\end{array}\right]
$$

If the Hamiltonian is invariant to translations in space, such that $\alpha=X$, the following expression for the pseudomomentum, $P$, is obtained:

$$
\Pi=P \equiv \frac{1}{2} \int_{-\infty}^{\infty} A B d X,
$$

The condition that the Hamiltonian be translationally invariant in the streamwise direction is equivalent to restricting attention to streamwise uniform flow. Thus the pseudomomentum is conserved for background flows that are streamwise uniform. Because the instability of streamwise uniform flow requires that the pseudomomentum vanish, a necessary condition for global mode instability in streamwise uniform flow is that the wave pair, $A$ and $B$, be orthogonal. Hence, the traditional supercritical instability condition of streamwise uniform flow that the background PV gradient must change sign may also be interpreted as the condition that physical/virtual wave 
pairs be orthogonal.

If the Hamiltonian is invariant to translations in time, such that $\alpha=T$, we obtain conservation of pseudoenergy $E$, where $\Pi=E \equiv-H$. It is instructive to partition the pseudoenergy into two components, one component for the physical wave $A$ and the other for the virtual wave $B: E=E_{A}+E_{B}$, where

$$
\begin{gathered}
E_{A}=\frac{1}{2} \int_{-\infty}^{\infty}\left[\left(\frac{\partial A}{\partial X}\right)^{2}-m_{3} A^{2}\right] d X, \\
E_{B}=\frac{1}{2} \int_{-\infty}^{\infty} B^{2} d X>0 .
\end{gathered}
$$

Each wave makes a distinct contribution to the pseudoenergy. Because $E_{B}>0$, instability requires that the physical wave have 'negative energy', i.e., the total pseudoenergy must vanish for instability [11]. Therefore, a necessary condition for instability is that $m_{3}>0$ somewhere in the flow. As discussed below, the condition $m_{3}>0$ leads to a supercritical dispersion relation, i.e., the $O(\epsilon)$ portion of the flow is such that it pushes the total flow across the marginal curve into the unstable region. Similarly, the condition $m_{3}<0$ results in both the physical and virtual waves having positive energy and therefore stability is assured. Thus, a sufficient condition for stability is $m_{3}<0$, which states that all globally subcritical Hamiltonian shear flows are stable, whereas instability requires a supercritical region $\left(m_{3}>0\right)$. In contrast, we will show in the next section that non-Hamiltonian shear flows may in fact be unstable even when no supercritical region exists in the flow. Lastly, note that the pseudoenergy of the physical wave has precisely the form of that for waves on deeply stable flows (see [13] and [14]).

In addition to the conservation of pseudomomentum and pseudoenergy, other conservation laws can be obtained by identifying the Casimir invariants $C$ that satisfy

$$
\mathbf{J}\left[\begin{array}{l}
\delta C / \delta A \\
\delta C / \delta B
\end{array}\right]=0
$$

One such Casimir invariant is "mass", which is given by

$$
M=\int_{-\infty}^{\infty}[A+B] d X .
$$

Because $M$ is conserved, the integrated sum of the coupled modes must vanish for instability. Because (3.23) states that the integrated amplitude of $A$ must vanish for instability, this implies that the integrated amplitude of $B$ must also vanish. Thus, like the physical wave, the virtual wave must have oscillatory structure.

\section{Non-Hamiltonian stability analysis}

Before carrying out the stability analysis for generalized non-Hamiltonian flow, it is instructive to first consider the case of streamwise uniform flow, for which $m_{3}$ is constant and $m_{5}=0$. Upon assuming the small-amplitude limit, (3.21) and (3.22) reduce to the linearized form of the classic Boussinesq equation, which yields the following quartic dispersion relation:

$$
D_{B}(k, \omega) \equiv \omega^{2}-k^{4}+m_{3} k^{2}=0 .
$$


As is well known, this dispersion relation may, depending on the parameter setting, describe linear exponential instability. We consider the local stability of the background flow for two different regimes: supercritical flow $\left(m_{3}>0\right)$ and subcritical flow $\left(m_{3}<0\right)$. Therefore, $m_{3}>0\left(m_{3}<0\right)$ occurs when the $O(\epsilon)$ portion of the flow, $U_{n}^{(1)}$, is such that it pushes the stability of the total flow across the marginal curve into the unstable (stable) region. Because we will employ flows in which $U_{n}^{(1)}$ is a function of the streamwise coordinate, $X$, we will be able to compare the instabilities that develop on locally subcritical and supercritical flows.

To provide context for the analytical (WKB) analysis to be presented below, and to preview the main differences between the unstable modes that develop on streamwise uniform and streamwise varying flows, we show in Fig. 5.1 generic plots of frequency and growth rate as a function of zonal wavenumber. For the streamwise uniform (Boussinesq) case (Fig. 5.1a), which is based on Equ. (5.1), there is a short wave cut-off and the unstable waves $(\operatorname{Im}(\omega)>0)$ have zero frequency. In sharp contrast, for the streamwise varying case (Fig. 5.1b), which is based on (5.6) below, there is no short wave cut-off and the unstable modes have non-zero frequency.
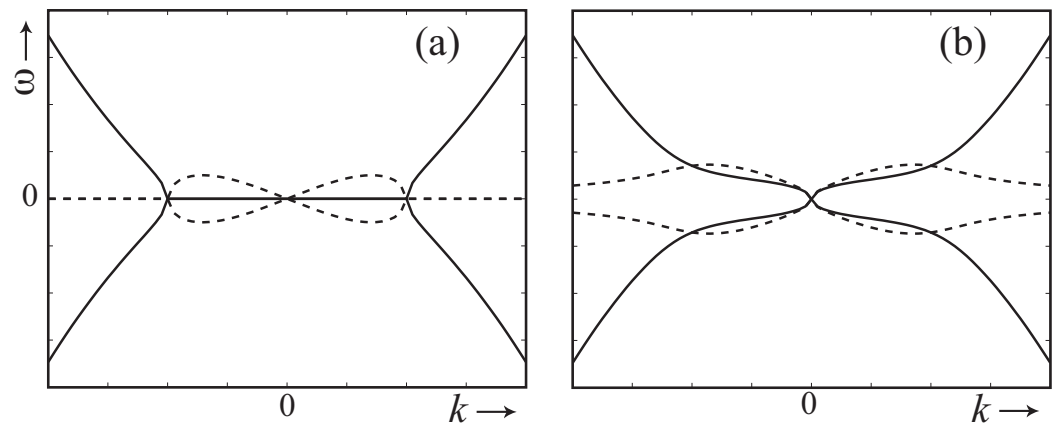

FIG. 5.1. Generic depiction of the variation of frequency versus wavenumber for (a) zonally uniform flow $\left(m_{5}=0\right)$, which derives from the linearized form of the classic Boussinesq equation, and $(b)$ streamwise varying flow $\left(m_{5} \neq 0\right)$. Solid lines denote Re $(\omega)$ and dashed lines denote $\operatorname{Im}(\omega)$.

5.1. WKB analysis. To obtain an analytical expression for the global mode frequency and growth rate, we assume that the streamwise variations in the background flow are 'slow' such that $\chi=\delta X$, where $\delta \ll 1$ measures the ratio of the long wave variation to that of the background flow. We seek solutions to (3.21) and (3.22) of the form

$$
\left[\begin{array}{l}
A \\
B
\end{array}\right]=\left[\begin{array}{l}
a(\chi) \\
b(\chi)
\end{array}\right] \exp \left[-i\left(\omega_{0}+\delta \omega_{1}+\ldots\right) t\right]+\text { c.c., }
$$

where the frequency $\omega_{j} \in \mathbb{C}$, and cc denotes the complex conjugate of the preceding term. Expanding the amplitude $a$ in standard WKB form [17],

$$
a(\chi)=\exp \left[\Theta_{0}\left(\chi ; \omega_{0}\right) / \delta+\Theta_{1}\left(\chi ; \omega_{1}\right)+\ldots\right],
$$

where $k_{0}\left(\chi, \omega_{0}\right)=-i d \Theta_{0} / d \chi$ is the local zonal wavenumber, leads to the following relationship between the physical and virtual wave amplitudes, $a$ and $b$ :

$$
\left[\begin{array}{l}
a \\
b
\end{array}\right]=\left[\begin{array}{c}
1 \\
-c_{0}
\end{array}\right] \exp \left[\Theta_{0}\left(\chi ; \omega_{0}\right) / \delta+\Theta_{1}\left(\chi ; \omega_{1}\right)+\ldots\right],
$$


where $c_{0}=\omega_{0} / k_{0} \in \mathbb{C}$ is the phase speed of the waves. $c_{0}$ also measures the ratio of the virtual to physical wave amplitudes, with the phase relationship between the waves given by

$$
\alpha=\tan ^{-1}\left(\frac{c_{i}}{c_{r}}\right),
$$

where $c_{0}=c_{r}+i c_{i}$. For $c_{0}=c_{r}$, the waves are $0^{\circ}$ or $180^{\circ}$ out of phase. If $c_{r}>0$, the wave pair has negative pseudomomentum, and if $c_{r}<0$ the wave pair has positive pseudomomentum. Recall that wave pairs with non-zero pseudomomentum must extract pseudomemontum from the background flow in order to grow (see Equ. (3.25)). For $c_{0}=c_{i}$, the physical and virtual waves are $90^{\circ}$ or $270^{\circ}$ out of phase and thus have zero pseudomomentum. In this case the phase relationship between the physical and virtual wave is such that they are unable to exploit the pseudomomentum source in (3.26).

To lowest order the waves must satisfy the following dispersion relation:

$$
D\left(k_{0}, \omega_{0} ; \chi\right) \equiv \omega_{0}^{2}-k_{0}^{4}+m_{3}(\chi) k_{0}^{2}-i m_{5}(\chi) k_{0}=0 .
$$

If the leading order frequency $\omega_{0}$ were known, we would need only solve (5.6) for the wavenumber, $k_{0}$, at each location in $\chi$. This would yield the WKB phase function, $\Theta_{0}$, and thus the leading order WKB approximation. Consequently, to complete the WKB approximation we must identify the leading order (complex) frequency. As shown below, this requires also determining (1) the location of the branch point, $\chi_{0}$, and (2) the complex wavenumber $k_{0}$ at the branch point. We thus have three unknowns $\left[\chi_{0}, \omega_{0}, k_{0}\left(\chi_{0}, \omega_{0}\right)\right]$ whose determination requires three conditions.

As in HN06, obtaining an expression for the leading order (complex) frequency hinges on identifying the branch points in the complex $\chi$ plane. Because constructing WKB approximations requires validity across the entire complex plane, the search for branch points cannot be naively limited to the real axis. Otherwise, solutions (instabilities) will be missed. Once the complex branch point is located we may follow the Stokes line back across the real axis to determine where two branches of the WKB approximation change from subdominant (dominant) to dominant (subdominant). The point where the Stokes line crosses the real axis will be used as the matching point for the two branches of the dispersion relation (e.g., [18], [19], [20]).

For the quartic dispersion relation (5.6), where $k_{0}, \chi \in \mathbb{C}$, a point $\chi_{0}$ that is a square root branch point is defined by

$$
\left.\frac{\partial D}{\partial k_{0}}\right|_{\chi=\chi_{0}}=0 .
$$

Cube root branch points or higher are possible, but they typically turn out to be unphysical (e.g., [17]; HN07). To close the system, we use the equation

$$
\frac{d D}{d \chi}=\frac{\partial D}{\partial \chi}+\frac{\partial D}{\partial k_{0}} \frac{\partial k_{0}}{\partial \chi}=0
$$

Evaluating this equation at the branch point, subsequently using (5.7), and assuming the wavenumber is continuous at $\chi_{0}$, one obtains

$$
\left.\frac{\partial D}{\partial \chi}\right|_{\chi=\chi_{0}}=0 .
$$


From expressions (5.6), (5.7), and (5.9), we obtain the following equation whose roots are the branch points:

$$
F_{b r}\left(\chi_{0}\right)=4\left(\frac{d m_{5}}{d \chi}\right)^{3}+2 m_{3} \frac{d m_{5}}{d \chi}\left(\frac{d m_{3}}{d \chi}\right)^{2}-m_{5}\left(\frac{d m_{3}}{d \chi}\right)^{3}=0 .
$$

Fig 5.2 shows the branch point configurations in the complex- $\chi$ plane for the locally supercritical (left panel) and globally subcritical (right panel) parameter settings of Section 5.2. These branch point configurations appear as either two double poles (left panel) or four single poles (one pair is shown in the right panel).

At the branch point the local wavenumber is

$$
k_{0}\left(\chi_{0}, \omega_{0}\right)=i \frac{d m_{5} / d \chi}{d m_{3} / d \chi}
$$

and the leading order approximation to the global mode frequency is

$$
\omega_{0}\left(\chi_{0}\right)^{2}=\left(\left[\frac{d m_{5} / d \chi}{d m_{3} / d \chi}\right]^{3}+m_{3} \frac{d m_{5} / d \chi}{d m_{3} / d \chi}-m_{5}\right) \frac{d m_{5} / d \chi}{d m_{3} / d \chi} .
$$

Because the wavenumber at the branch point is complex, we expect spatial growth/decay of the solution across the branch point. Armed with the branch point location and the global mode frequency, we may now examine the solutions to (3.16) and (3.17).
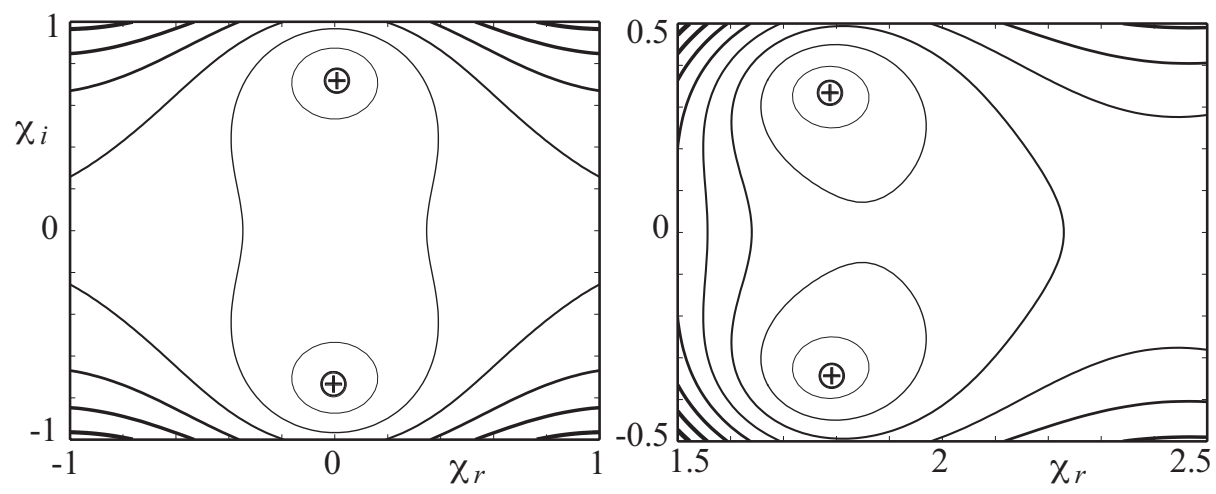

FIG. 5.2. Contours of $F_{b r}$ in the complex $\chi$-plane for locally supercritical flow (left panel) and globally subcritical flow (right panel). The $\oplus$ denotes the locations of the roots of $F_{b r}$. The locations of these roots are the branch points, which denote the location where a branch of the WKB approximation changes from subdominant to dominant.

5.2. Long Rossby wave solutions. The effects of streamwise variations on the growth and structure of long Rossby waves is examined for two cases that illustrate the general behaviors possible in the system. The first case is for locally supercritical flow and the second case is for globally subcritical flow. We show below that for locally supercritical flow the modes grow via wave-resonance, which is the same growth mechanism that occurs in streamwise uniform flow. In contrast, for globally subcritical flow, the modes grow via pseudomomentum extraction, the same 
growth mechanism that occurs in deeply stable streamwise varying flow.

In both the supercritical and subcritical cases we assume Gaussian variation in the background flow, which is perhaps the simplest way to represent streamwise variations. To simplify the coefficients in (3.20), we choose

$$
\begin{gathered}
m_{3}=-1+\alpha e^{-\chi^{2} / 9}, \\
m_{5}=4 \chi / 9 e^{-\chi^{2} / 9}
\end{gathered}
$$

where the free parameter $\alpha \in \mathbb{R}$ is chosen such that the flow is either globally subcritical $\left(m_{3}<0\right)$ or locally supercritical $\left(m_{3}>0\right)$. The relationship between the coefficients has been chosen to satisfy the constraint that the background flow must be horizontally non-divergent. The WKB parameter $\delta$ will be set to 0.2 .

Given the coefficients, the branch points and corresponding global mode frequencies can be calculated. Once the global mode frequencies are known, the dispersion relation (5.6) can be evaluated to determine the local wavenumber, $k_{0}$, and subsequently the WKB phase function, $\Theta_{0}$.

The left panel of Fig. 5.2 shows, for $\alpha=3$, a typical spatial configuration of the complex branch points that exist for locally supercritical flow. The branch points are off the real $\chi$ axis and in the locally unstable region $\left(m_{3}>0\right)$. In contrast, the right panel of Fig. 5.2 shows, for $\alpha=1$, a typical spatial configuration of the complex branch points that exist for globally subcritical flow. Again, the branch points are off the real $\chi$ axis, but now they are on the downstream side of the Gaussian well, i.e., in a streamwise shear zone where $d m_{3} / d \chi$ is large. The mirror image of this branch point configuration exists for $\chi_{r}<0$, which represents another mode attached to the streamwise shear zone on the upstream side of the Gaussian well.

Because the dispersion relation (5.6) is quadratic in $\omega_{0}$, there exists a pair of
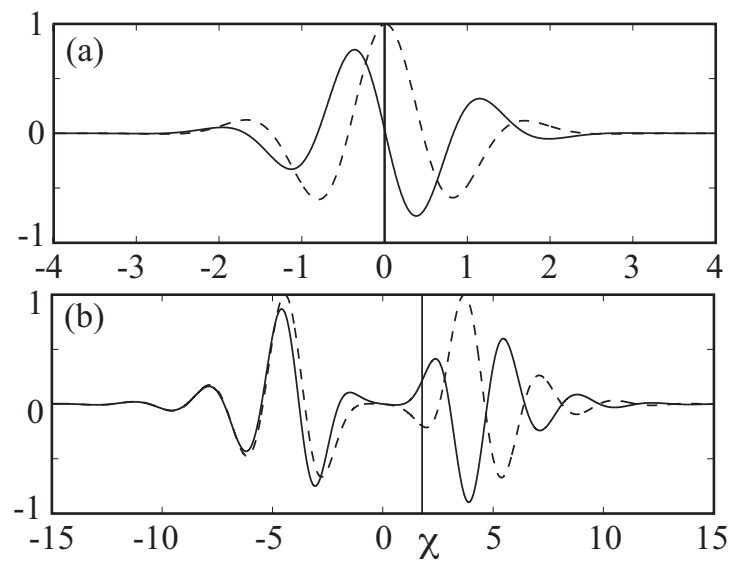

FIG. 5.3. The global mode structures for the physical wave (solid line) and virtual wave (dashed line). The vertical line denotes the projection of the branch point on the real axis. (a) Locally supercritical flow; (b) globally subcritical flow.

complex conjugate modes at each branch point. Focusing our attention on the unstable supercritical modes of Fig. 5.2, both branch points are associated with equal growth rates, $\operatorname{Im}\left(\omega_{0}\right)=0.92$, and zero frequencies, $\operatorname{Re}\left(\omega_{0}\right)=0$. For subcritical flow, 
the unstable modes (right panel, Fig. 5.2) have opposite frequencies: $\operatorname{Re}\left(\omega_{0}\right)=-0.39$ for the point where $\chi_{i}>0$, and $\operatorname{Re}\left(\omega_{0}\right)=0.39$ for the point where $\chi_{i}<0$. However, both branch points have equal growth rates, $\operatorname{Im}\left(\omega_{0}\right)=0.15$. In addition, there exists two additional modes attached to the mirror image of the branch point configuration in the right panel of Fig. 5.2, i.e., for $\chi_{r}<0$. These modes have the opposite real frequency arrangement about the $\chi_{r}$-axis, but the same growth rates as the pair of modes for $\chi_{r}>0$. This suggests that the global mode in subcritical flow is constructed from a quartet of growing, counter-propagating physical waves with equal growth rate.

Fig 5.3 shows the lowest order approximation for the wave structure. For locally supercritical flow (Fig. 5.3a), the wave is strongly trapped to the Gaussian well. Because (5.6) is quartic in wavenumber, $k_{0}$, the solution obtains four branches. A careful examination of these branches shows that only certain pairs can be matched that satisfy the streamwise boundary conditions. Thus two solutions are created that satisfy the boundary conditions. In the locally supercritical case, the two solutions are identical. We also note that the WKB solutions are robust; that is, they remain valid beyond their strict range of validity, which corresponds to $\delta \ll 1$. In particular, although we have used $\delta=0.2$ in order to formally conform to WKB scaling, the structure of the supercritical instabilities under this WKB scaling compare favorably to solutions obtained by HN04 with $\delta=1$. The WKB solutions were also found to be robust in the deeply stable case [14].

In contrast, the structure of the globally subcritical instabilities are such that the matched pairs are anchored on either side of the Gaussian well (see Fig. 5.3b). Both wave pairs have been plotted by assigning them unit amplitude and then summing. The solution shown in Fig 5.3b exhibits significant penetration of Rossby wave radiation into the far-field. The penetration into the far-field was found to be controlled by the sign of $m_{5}$ in (3.10), i.e., if a minus sign is arbitrarily added to the right-hand side of (5.14), the penetration into the far-field is eliminated and the wave structure is confined to the Gaussian well.

In Fig. 5.3, for the subcritical modes, the physical/virtual wave pair, $A$ and $B$, appear nearly anti-parallel downstream of the Gaussian variation, but almost parallel upstream. For the supercritical mode, the physical/virtual wave pair appear orthogonal. These phase relationships, which are based on visual inspection of Fig. 5.3, were verified by explicitly calculating the pseudomomentum for the modes. The supercritical mode has $P=0$, which confirms that the mode on the locally supercritical flow must be growing through wave-resonance. In contrast, the upstream (downstream) mode in Fig. 5.3b has $P=1.5(P=-1.3)$, which is consistent with the pseudomomentum arguments given earlier based on (3.25).

\section{Conclusions}

The stability of streamwise varying shear flow that is marginally stable to long Rossby waves has been examined. Departures from marginal stability are due to local streamwise variations in the flow. Constraints on allowable wave structures and general stability criteria have been obtained. We have shown using a coupled wave instability (CWI) framework that all exponentially amplifying disturbances must have oscillatory structures. In sharp contrast, exponentially amplifying disturbances that develop on streamwise varying shear flow that is deeply stable can have either oscillatory or non-oscillatory structures [14].

Equations for pseudoenergy and pseudomomentum were derived and used to obtain general stability properties. We proved that all globally subcritical Hamiltonian shear flows are stable and that instability requires a region in the flow that is locally 
supercritical. On the other hand, we have used the fact that pseudomentum is not conserved for either Hamiltonian or non-Hamiltonian streamwise varying shear flow in order to provide conditions for classifying the growth mechanism, predicting the regions along the flow where the waves can grow, and predicting the structural characteristics of the waves.

A WKB analysis was applied to two distinct types of non-Hamiltonian background flows: (1) locally supercritical flow, which operates like an unstable streamwise uniform flow that is simply bounded on either side by stable flow; and (2) globally subcritical flow. For locally supercritical flow, the classic integral theorem for instability, which requires that the flow possess an inflection point somewhere in the domain, is satisfied within the supercritical flow region (see Equ. (3.5)). We have shown that in locally supercritical flow, an unstable disturbance consists of a physical/virtual wave pair that is highly localized to the locally unstable region. The growth mechanism in this locally supercritical case was shown to be wave-resonance, which is the traditional mechanism for disturbance growth in streamwise uniform flow. Thus, an alternative interpretation of the classic integral theorem for instability is as the condition for wave resonance between a physical/virtual wave pair.

For globally subcritical flow, the integral theorem for instability is not satisfied at any streamwise location in the flow. Nevertheless, we have found instabilities on non-Hamiltonian subcritical flows. The existence of these instabilities highlights the distinction between the local and global stability properties of streamwise varying shear flows. Moreover, the growth mechanism for modes on subcritical flow was shown to be distinctly different from traditional wave-resonance. The growth mechanism on globally subcritical flow is characterized by pseudomomentum extraction from the streamwise variations in the flow. These subcritical instabilities consist of coupled waves composed of physical/virtual wave pairs that are parallel or anti-parallel depending on the local properties of the local pseudomomentum source.

Acknowledgement. This research was performed while D. Hodyss held a National Research Council Research Associateship Award at the Naval Research Laboratory. Funding for T. Nathan was provided by NASA's Living with a Star, Targeted Research and Technology Program, Grant LWS04-0025-0108.

Appendix A. Cross-stream structure functions. An analytical solution for the cross-stream $(y)$ structure of the unstable waves can be obtained by assuming that to lowest order the streamwise uniform background flow satisfies

$$
Q_{n y}^{(0)}=-\kappa_{n} U_{n}^{(0)}
$$

where $\kappa_{n}$ is a (possibly different) constant in each layer. $U_{n}^{0}=$ constant is an example of a background flow that satisfies A.1. The solution to (3.8), with $\kappa_{n}$ a constant, is

$$
H_{n}=c_{n} \sin (l y)+d_{n} \cos (l y)
$$

where the $c_{n}$ and $d_{n}$ are constants. The cross-stream wavenumber, $l$, must satisfy

$$
l^{2}=-\left(F+\frac{\kappa_{1}+\kappa_{2}}{2}\right) \pm \sqrt{F^{2}+\left(\frac{\kappa_{1}+\kappa_{2}}{2}\right)^{2}-\kappa_{1} \kappa_{2}} .
$$

Because we require $l \in \mathbb{R}$, it follows that $\kappa_{1} \kappa_{2}<0$. The only way to achieve this is for the background potential vorticity gradient in each layer to have opposite 
sign. This latter condition is simply a manifestation of the necessary condition for instability for streamwise uniform flow in the (quasigeostrophic) two-layer model ([12]). This connection between the cross-stream wavenumber $l$ and the satisfaction of this stability condition could have been predicted from our starting assumption that we would focus on flows that are marginally stable.

Appendix B. Coefficients in the amplitude Equ. (3.9).

$$
\begin{gathered}
m_{1}=-\sum_{n=1}^{2} \int_{-1}^{1} \kappa_{n}\left(f_{n}-\frac{H_{n}}{U_{n}^{(0)}}\right)^{2} d y \\
m_{2}=\sum_{n=1}^{2} \int_{-1}^{1} H_{n}^{2} d y, \\
m_{3}=\sum_{n=1}^{2} \int_{-1}^{1}\left[\kappa_{n} U_{n}^{(1)}+Q_{n y}^{(1)}\right] \frac{H_{n}^{2}}{U_{n}^{(0)}} d y, \\
m_{5}=\sum_{n=1}^{2} \int_{-1}^{1}\left[V_{n}^{(1)}\left(\kappa_{n} H_{n}\right)_{y}+Q_{n X}^{(1)} H_{n y}\right] \frac{H_{n}}{U_{n}^{(0)}} d y,
\end{gathered}
$$

where $f_{n}(y)$ and $H_{n}(y)$ are related by

$$
f_{n y y}+F(-1)^{n}\left(f_{1}-f_{2}\right)-\kappa_{n} f_{n}=-\frac{\kappa_{n}}{U_{n}^{(0)}} H_{n} .
$$

In B.1-B.5 the background PV gradients are given by

$$
\begin{aligned}
& Q_{n y}^{(1)}=-U_{n y y}^{(1)}-F(-1)^{n}\left(U_{1}^{(1)}-U_{2}^{(1)}\right), \\
& Q_{n X}^{(1)}=-V_{n y y}^{(1)}-F(-1)^{n}\left(V_{1}^{(1)}-V_{2}^{(1)}\right) .
\end{aligned}
$$




\section{REFERENCES}

[1] L.J. Campbell, Wave-mean-flow interactions in a forced rossby wave packet critical layer, Studies in Applied Mathematics, 112, 39-85, 2004.

[2] J. Vanneste, A nonlinear critical layer generated by the interaction of free Rossby waves, J. Fluid Mech., 371, 319-344, 1998.

[3] E. Tikhomolov, Forcing of differential rotation and Rossby waves at the interface between the convectively stable and unstable layers, Astrophys. Journal, 499, 905-913, 1998.

[4] J.R. Kuhn, J.D. Armstrong, R.I. Bush and P. Scherrer, Rossby waves on the Sun as revealed by solar 'hills', Nature, 405, 544-546, 2000.

[5] R.E. Dickinson, Quasi-geostrophic motions in a galactic flow, PAGEOPH, 59, 155-171, 1964.

[6] M. Tagger and F. Melia, A possible Rossby wave instability origin for the flares in Sagittarius A, The Astrophysical Journal, 636(2), 33-36, 2006

[7] K.R. Helfrich and J. Pedlosky, Time-dependent isolated anomalies in zonal flows, J. Fluid Mech., 93, 377-409, 1993.

[8] K.R. Helfrich and J. Pedlosky, Large-amplitude coherent anomalies in baroclinic zonal flows, J. Atmos. Sci., 52, 1615-1629, 1995.

9] L.O. Merkine, The stability of quasigeostrophic fields induced by potential vorticity sources, J. Fluid Mech., 116, 315-342, 1982.

[10] D. Hodyss and T.R. Nathan, The connection between coherent structures and low frequency wave packets in large-scale atmospheric flow, J. Atmos. Sci., 61, 2616-2626, 2004.

[11] R.A. Cairns, The role of negative energy waves in some instabilities of parallel flows, J. Fluid Mech., 92, 1-14, 1979.

[12] J. Pedlosky, Geophysical Fluid Dynamics, Springer, 710, 1987.

[13] D. Hodyss and T.R. Nathan, Instability of variable media to long waves with odd dispersion relations, Commun. Math. Sci., 4(3), 669-676, 2006.

[14] D. Hodyss and T.R. Nathan, The role of forcing in the local stability of stationary long waves, Part 1. Linear dynamics, J. Fluid Mech., 576, 349-376, 2007.

[15] P. Stone, Baroclinic adjustment, J. Atmos. Sci., 35, 561-571, 1978.

[16] D. Hodyss and T.R. Nathan, Long waves in streamwise varying shear flows: new mechanisms for a weakly nonlinear instability, Phys. Rev. Lett., 93(7): Art. No. 074502 Aug 13, 2004.

[17] C.M. Bender S. and A. Orszag, Advanced Mathematical Methods for Scientists and Engineers, McGraw Hill, 1978.

[18] J.P. Boyd, Weakly Nonlocal Solitary Waves and Beyond-All-Orders Asymptotics, Kluwer Academic, 1998.

[19] S. Le Dizes, P. Huerre, J.M. Chomaz and P.A. Monkewitz, Linear global modes in spatially developing media, Phil. Trans. R. Soc. Lond. A, 354, 169-212, 1996.

[20] P. Huerre and M. Rossi, Hydrodynamic instabilities in open flows, Hydrodynamics and Nonlinear Instabilities, C. Goldrèche and P. Manneville Ed., Cambridge University Press, 81-294, 1988. 